\title{
LEPROSY IN THE BENIN AND WARRI AREAS OF NIGERIA \\ L. LENGAUER
}

The Benin and Warri Provinces are situated on the delta of the river Niger, and therefore lie on the border of swamp ground and dense forest. The land, as would be expected, is low lying and the climate is hot and humid.

This area is sparsely populated. In contrast to the more 
eastern provinces of Nigeria, the percentage of infective cases is large. This may be related to the large number of lepromatous children observed in the area.

A curious feature of the lepromatous type of leprosy in these provinces is the high incidence of actual nodular involvement. For example, a recent examination of a segregation village shewed one hundred infectious cases. Of these fifty-three were nodular, ten shewed diffuse infiltrative lepromata, twenty-nine were a mixed " unclassified " but positive group, while eight were tuberculoid evolving towards lepromatous.

The case histories of lepromatous patients here would seem to sugge'st that a small number of patients record nodules as the first sign of the onset of the disease. Many patients give a history of an ulcer of the foot as a primary lesion. The majority, however, state that what they notice first is a single depigmented macule. In most of these cases, after a relatively short space of time, a fresh crop of ill defined macules appear. Only at a much later stage is there lepromatous infiltration in the form of nodules. The crop of vague macules is not an essential feature: in many cases this phase appears to be omitted. In some cases the early macule displays definite clinical characteristics of the tuberculoid type, which may persist from one to three years without significant change.

I have observed a number of cases in which all three of the above phenomena have been observed at the same time, i.e., macules with no characteristic feature beyond depigmentation, tuberculoid lesions and lepromatous nodular infiltration. This sequence is observed in the number of cases examined in Ossiomo Leper Settlement which have been graded as tuberculoid or of indefinite macular structure and bacteriologically negative on admission, and which, after one or two years, have become positive with diffuse infiltrative lepromatous lesions and nodules.

The evolution from tuberculoid to lepromatous leprosy is rarely associated with lepra reaction or with any acute phase of the disease. Puberty however appears to be a critical time. In females a marked deterioration of the leprotic condition is frequently observed here during pregnancy, and particularly during the period of lactation. This tendency towards evolution from tuberculoid to lepromatous leprosy can be arrested as a result of treatment. The change for example is much less frequent at the Settlement and in the Segregation Villages than it is amcng out-patients.

Bacteriologically positive and lepromatous cases:- These may be classified generally as infectious forms, and they display a considerable variety of lesions. The cases which are apparently 
nodular from the start shew little sign of change and remain static. Major tuberculoid lesions, particularly those which are bacteriologically positive, shew a definite tendency towards lepromatous evolution, and eventually nodular formation.

Early lepromatous cases are often characterised by a faint diffuse blush with no definite edge, on the upper part of the back towards the shoulders. This is not easy to see on the African skin, and may be missed unless the angle of light is favourable. These lesions are usually extremely rich in bacilli and tend to spread rapidly. A curious feature is that frequently the trunk lesions, which are difficult to detect, are accompanied by gross nodular formation on the face and ears.

The difficulty in diagnosis, however, is illustrated by one case at Ossiomo Leper Settlement with an apparently perfectly normal skin. On examination the only abnormalities found were a tiny ulcer at the outer side of the left ankle and some anæsthesia of the fect. Smears were taken from the ear lobe as no lesions on the body were visible. The smears were found to contain $\mathbf{M}$. leprae in very large numbers. The subsequent history of this case is instructive. He left the Settlement and reappeared several years later with signs of pleurisy and commencing pulmonary tuberculosis. Even at this stage thickening of the ears and faint infiltration of the face could be detected only after careful examination. Smears taken from the ear and back were highly positive. Yet this man had for years escaped notice among a healthy population while in an infective state.

Nodular formation is not infrequent here round the mouth and alae nasi. These sometimes ulcerate and later produce fibrous contraction. In late cases with extensive involvement, nodules are found not only on the face, but on the arms, legs and chest. An interesting and unusual feature of advanced leprosy here is the appearance of ulcerating nodules on the male genitalia (I have never seen nodules on the vulva). Another common condition (which appears to be unrecorded elsewhere) is sten urinary meatus in advanced lepromatous males. The meatus is in some cases reduced to a pin point opening, requiring artificial dilatation.

Leprotic blindness is rare in this area. Out of some five hundred lepromatous cases at Ossiomo Settlement only three are blind. Lepromatous ulceration is also comparatively rare and responds well to treatment.

Pyorrhoea is a very frequent complication of lepromatous cases here. This condition is important both from its resistance to treatment and its lowering effect on the general health of the 
patient. Laryngitis is extremely common among lepromatous cases with extensive lesions.

Among lepromatous outpatients from the bush, anæmia with cardiac complications is often marked. Here I am inclined to think that leprotic anæmia is only one of several etiological factors. Albuminuria is also frequent among our lepromatous cases. This demands very careful regulation of hydnocarpus oil treatment and sometimes cessation of medication.

Among unusual complications it may be worth while mentioning tenosynovitis-ari acute and painful condition occurring during reaction.

Death as a result of reaction is so infrequent that three cases here are worthy of record. All three cases followed the same course. They started with lepromatous reaction, rash and slight rise of temperature. In each case the patient complained of generalised pain and, more especially, pain of the feet. The urine contained a little albumen. The total quantity of urine per twenty-four hours became reduced to 6 or 7 ounces. This was followed by generalised pruritis and convulsions, and later the patients became delirious. The urinary output fell to 3-4 ounces per twenty-four hours and was unaffected by the administration of diuretics. Death occurred in each case after 7-Io days' illness. Unfortunately it was not possible to study these cases in greater detail, and post-mortem findings could not be obtained owing to lack of facilities.

Lepra reaction is infrequent here. Occasionally one observes repeatedly recurring lepra reaction in the same patient, an unfortunate condition for which general health rehabilitation appears to be the only treatment.

Non-infectious cases. In this area, as compared with other parts of West Africa, the proportion of tuberculoid cases among the non-infectious group is relatively large. An examination of a new village lately shewed the following fairly typical proportion:-

$\begin{array}{lccccccr}\text { Tuberculoid } & \ldots & \ldots & \ldots & \ldots & \ldots & \ldots & \text { II } 2 \\ \text { Macular } & \ldots & \ldots & \ldots & \ldots & \ldots & \ldots & 68 \\ \text { Macular-Tuberculoid } & \ldots & \ldots & \ldots & \ldots & \ldots & 5 \\ \\ \text { Total number of non-infectious patients } \\ \text { examined } & \text { I85 }\end{array}$

Vague ill-defined multiple macules frequently appear in crops, mainly on the back. These appear to be frequent in cases of relapse. In one case I have seen such macules disappear after several months without treatment of any kind. 
Tuberculoid cases shew erythematous, sometimes scaly lesions with well-defined margins and slightly raised thickened edges. The size and number of these legions vary considerably.

I have not infrequently observed one or two large widespread tuberculoid lesions accompanied in the same patient by widely disseminated small tuberculoid patches. In connection with this I often see large tuberculoid lesions with small independent satellites very close to the margin of what I have termed the " mother macule." Single tuberculoid lesions of any size are rare. One most remarkable feature. of such cases is that they tend to come from the same locality. For instance, Orogun village has a considerable number of such cases, while the village next to it has none. In one case I observed a single macule which remained as an isolated lesion for twenty years. A considerable crop of macules then appeared. This, however, is in my experience exceptional.

I have seen one case of an infant with clinical leprosy. The parents were both non-infectious lepers, but it is possible that the child may have been in contact with open cases. The first lesion appeared on the back when the child was seven months old. This was followed by a number of vague small irregular, rather hypopigmented lesions. The child was given treatment with hydnocarpus oil and the lesions disappeared. She is now eight years old and no further signs of the disease have developed.

Both in typical tuberculoid cases and in those with the strictly macular leprosy so common in Nigeria, there is usually a lapse of time of one to three months between the appearance of the first lesion and the outbreak of a crop of new lesions.

I have seen only three cases of ulcerative tuberculoid leprosy. In these cases the lesions were near the edge of the eye, and the eye in one case was lost through secondary infection. Occasionally one encounters tuberculoid lesions (not major) which are bacteriologically positive. This, however, is rare.

Localisation. In most cases in this area lesions are multiple and widely spread. The prognosis, however, is distinctly more hopeful in those cases with tuberculoid lesions on the forehead, elbows and knees, than in those with isolated lesions with very raised edges on the arms or single "tennis racquet" lesions on the back. On the other hand, the prognosis seems bad in cases where there are enormous macules covering the whole of the face or back. In the macular leprosy of this area tactile anæsthesia is uncommon in early cases, and is frequently not elicited even in advanced cases.

I have never personally observed the process of self-healing 
in leprosy, although I have heard from patients stories of spontaneous cure. I examined recently a woman with infiltrated skin of the face accompanied by a curious pallor-the appearance being extremely suggestive of lepromatous leprosy. Repeated bacteriological examinations failed to reveal bacilli. The woman stated that she was " very sick with leprosy" during pregnancy and after confinement, but was much improved now-her child is four months old. This apparently spontaneous improvement seems to contradict my experience.

In macular cases very often the first manifestation of leprosy is neural, often characterised by pain in the foot, or the appearance of an ulcer. Only after that do the macules appear.

There is little variation in the appearance of these unclassified macules. The word "vague" is probably the most suitable for their description. The macules may be single or few in number to begin with. These vague flat lesions are never in my experience bacteriologically postive. They are sometimes difficult to see and must be examined in a good light, and at the proper angle. They may appear on the limbs or back, but frequently they are widely spread over the body surface and tend to coalesce. The margins of such macules are often of the " geographical map " appearance, protruding here, receding there. These macules must be differentiated from the slightly or uniformly raised lesions which have sometimes a velvety appearance, and are extremely rich in bacilli.

In all types of cases the statement is frequently made that, previous to any spread or exacerbation of the lesions, there is an itching or creeping sensation all over the body. This prodromal itching may last several weeks.

Cases with tuberculoid leprosy usually shew better general health than the unclassified macular group. In the unclassified macular group the main nerves are more rapidly involved.

Mutilation of the hands and feet is extremely prevalent in all types of cases. The high percentage of mutilation, claw hand, digital loss, etc., can be explained by the frequent evolution of unclassified and tuberculoid cases towards the lepromatous stage.

The non-infectious forms of leprosy here are exceedingly prone to early ulceration. These ulcers if unattended become quickly septic. The majority of deaths among untended lepers is caused by sepsis. Mutilations are frequent and sometimes occur very suddenly. They are caused by gangrene which is often due to traumatism. In Ossiomo Settlement, since I934, we can still count on our fingers the fatal cases of gangrene, butt extensive and rapid destruction of tissue of the hands and feet is not uncommon, 
There are quite a number of cases of facial paralysis affecting the muscles of the eyes and mouth. In such cases the skin lesion accompanying the nerve involvement is hardly visible, but covers the whole face.

In this area I have only seen one case of leprotic alopæcia.

Another characteristic of leprosy in this area is the tendency to rapid progress of the disease and quick change of type. This renders medical examination, classification and assessment extremely difficult. For instance, it is not uncommon to see a patient who has kept his primary lesion-an unclassified macule or a tuberculoid area-but is now nodular and bacteriologically positive. I have seen patients under treatment shewing (a) residual tuberculoid lesions which have subsided under treatment; (b) typical tuberculoid lesions unaffected by treatment, and (c) a fresh crop of unclassified macules. Again nodular cases may develop a few new unclassified macules which are bacteriologically negative.

Other patients, particularly those who have not received treatment, often shew erythematous scaly tuberculoid lesions and, at the same time, thickened lepromatous ears and infiltrated skin of the face bacteriologically positive. There may be an amazing variety of heterogeneous macules and leprotic lesions on the same patient. It frequently happens that a patient is accurately charted and classified, only to shew a few months later a bewildering new set of lesions which in no way correspond with the original description.

Conclusions:

The rapid evolution of types and complications seen in this area provide excellent material for research. Some of the vagaries of each type are described. 